\title{
Adsorption and Mobility of Lithium on Pristine and Stone-Thrower-Wales Defective Silicenes
}

\author{
Yan Xin and Yang-Xin $\mathrm{Yu}^{*}$ \\ Laboratory of Chemical Engineering Thermodynamics \\ Department of Chemical Engineering, Tsinghua University \\ Beijing 100084, P. R. China \\ e-mail:yangxyu@mail.tsinghua.edu.cn
}

\begin{abstract}
To explore and rationalize the enhancement of the capacity of lithium-ion batteries, ab initio density functional theory was employed to investigate the adsorption and diffusion properties of a lithium atom on and through pristine and Stone-Thrower-Wales (STW)-defective silicenes. The theoretical calculations indicate that an enhancement of binding energy for a lithium atom on defective silicenes is found and the adsorption on pristine and defective silicenes is stronger than those on their graphene counterpart. The lithium mobility on pristine and defective silicenes is comparable to those on their graphene analogue, but the lithium atom migrates more easily and fast through the pores in silicene nanosheets than through the pores in corresponding graphene nanosheets. In most cases, van der Waals (vdW) interactions contribute $0.78-0.99 \mathrm{eV}$ to the binding energies. They can cause either an increase or a decrease in diffusion barrier dependent on the competition of their contributions to the energies between the transition state and initial structures. The vdW interactions are dominant for adsorption, but some time their effects on diffusion barrier are only marginal due to that their contributions may cancel each other out.
\end{abstract}

Keywords-silicene; STW defect; adsorption; mobility; diffusion barrier

\section{INTRODUCTION}

Silicon-based anodes have a theoretical capacity of $4200 \mathrm{mAh} / \mathrm{g}$, which is much higher than that of graphite $(372 \mathrm{mAh} / \mathrm{g})$. However, the bulk crystalline silicon anodes suffer from huge volume changes upon lithiation [1], leading to fracture of the electrode and then a slower charge rate [1, 2]. These issues limit the commercialization of the siliconbased battery technology [3].

Recent advances in two-dimensional layered materials have offered an opportunity to develop alternatives to the bulk silicon-based anode. Great success has been achieved in this area for graphene in both its defects and doped structures as anode materials [4]. As an analogue to graphene, silicene has been considered to serve as a promising high-capacity anode of lithium-ion batteries [5]. The adsorption characteristics of lithium atoms on a silicene nanosheet were explored by Osborn et al. [6] using ab initio density functional theory and molecular dynamics simulation. They found that the fully lithiated silicene was formed to be the most thermodynamically stable state of the $\mathrm{Li}-\mathrm{Si}$ compounds. Theoretical investigation of Tritsaris et al. [7] showed that the energy barriers for lithium diffusion are typically less than $0.6 \mathrm{eV}$. Furthermore, the volume only increases by $13 \%$ and $24 \%$ for full lithiation of the monolayer and bilayer silicenes, respectively. They can restore to their original structures after a complete delithiation. These features indicate that the freestanding monolayer or bilayer silicenes could be promising electrode materials with high energy density and long cycle life.

During the synthesis of two-dimensional materials various types of topological defects such as vacancies are quite likely to be created. Sahin et al. [8] have demonstrated theoretically that the energy barrier to form Stone-Wales (SW) defects in freestanding silicene is substantially lower than that in graphene. Similar to graphene, the defects in silicene are expected to have a significant influence on the mobility and adsorption energy of a silicene anode due to their fast pathway for diffusion and large room for storage.

To provide useful guidance on the development of new anode materials with excellent performance, a comprehensive understanding of defect effect on lithium adsorption and diffusion is essential. Ab initio DFT is a powerful tool to understand the interaction between the lithium atoms and the surface of the silicene defects. However, the use of DFT with the generalized gradient approximation (GGA) becomes problematic for the systems where van der Waals (vdW) interactions are significant because the GGA kernel completely lacks attractive dispersive or vdW interactions. Since high-level theories constructed by including non-local vdW interactions are still totally impractical for large systems, the addition of computationally efficient semiempirical dispersion correction to the semilocal DFT is a sensible alternative. In this method, vdW interactions are approximated by a pairwise inter-atomic $\mathrm{C}_{6} \mathrm{R}^{-6}$ term multiplied by a damping function [9]. A previous work [10] of a lithium atom adsorbed on monolayer and bilayer graphenylenes showed that $\mathrm{vdW}$ interactions contribute $14-32 \%$ attractive interactions to the adsorption energy. As we know, no detailed investigation was reported to evaluate how much the $\mathrm{vdW}$ interactions contribute to the diffusion barrier of a lithium atom on a two-dimensional layered material like graphene or silicene.

In view of this, the potential applications of defective silicene in lithium-ion batteries are explored and rationalized 
in this work. Ab initio DFT with dispersion correction (DFTD) [9] was employed to investigate how the vdW interactions influence the binding energy and diffusion barriers of a lithium atom on silicene. The presence of the Stone-Thrower-Wales (STW) defect in silicene was considered. The lithium atom adsorption on various sites and barriers of all possible diffusion paths on the defective silicenes were investigated in detail. It is found that a lithium ion can easily diffuse on the surface of silicenes studied and through the STW defect, which is of benefit to the charge/discharge performance of lithium-ion batteries.

\section{Computational Details}

In the present work, adsorption and diffusion of a lithium atom on the defective silicenes were investigated using $a b$ initio DFT implemented in the $\mathrm{DMol}^{3}$ code $[11,12]$. The exchange and correlation functional were treated by the GGA in the Perdew-Burke-Ernzerhof (PBE) version with spin polarization. Double numeric polarized (DNP) basis sets in version 4.4 were selected and the core electrons were treated using all electron relativistic method with a global orbital cutoff radius of $5.6 \AA$ for all elements. The Fermi smearing with a width of $0.14 \mathrm{eV}$ was used. The dispersion corrections of Grimme (DFT-D) [10] were also included in the calculation. Geometric structures of the systems studied were fully relaxed by minimizing the forces on each atom and the total energy change to below $5.4 \times 10^{-3} \mathrm{eV} / \AA$ and $3 \times 10^{-5} \mathrm{eV}$, respectively. The silicene substrate was modeled as a sheet with a $6 \times 6$ supercell. A 40 -Angstron-thick vacuum layer was placed between the sheets in the direction normal to the sheets. An $8 \times 8 \times 1 \mathrm{k}$-point sampling scheme was used to get the required accuracy. The charge of an adsorbed lithium atom was obtained by performing the Hirshfeld analysis. A refined $12 \times 12 \times 1$ Monkhorst-Pack grid was use in the calculation of the density of states (DOS).

The formation energy ( $\Delta E_{f}$ ) for each defective silicene was obtained by

$$
\Delta E_{f}=E_{\text {defect }}+n \mu_{\mathrm{Si}}-E_{\text {pristine }}
$$

where $E_{\text {defect }}$ is the energy of the defective silicene, $E_{\text {pristine }}$ is the energy of the pristine silicene, $\mu_{\mathrm{Si}}$ is the energy per atom for the pristine silicene, and $n$ is the number of silicon atoms removed from the pristine silicene to form the defective structures.

The binding energy $\left(E_{\mathrm{b}}\right)$ of a lithium atom with the pristine and defective silicene substrates were given by

$$
E_{b}=\left(E_{\text {defect }}+E_{\mathrm{Li}}\right)-E_{\mathrm{Li}+\text { defect }}
$$

where $E_{\mathrm{Li}+\text { defect }}$ is the total energy of the adsorption system of the silicene substrate and the adsorbed lithium atom and $E_{\mathrm{Li}}$ is the energy of one lithium atom in the stable bcc crystal.

In searching the diffusion transition state (TS), a linear synchronous transit (LST) was carried out and the conjugated gradient minimizations and quadratic synchronous transit (QST) maximizations were then repeated until a TS structure has been determined. All determined TS structures were validated and the diffusion paths were found using the nudged-elastic-band method [13]. The diffusion barrier $\left(E_{\text {act }}\right)$ is defined as the energy difference between the
TS structure and the given initial structure. According to the Arrhenius equation, the difference in diffusion barrier causes changes in the diffusion coefficient. Thus only diffusion barriers were calculated in this work since we focus on the improvement of ion mobility.

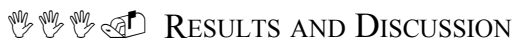

\section{A. Pristine and STW-Defective Silicenes}

The fully relaxed pristine silicene forms a buckled hexagonal structure with two sublattices located in different positions in the direction normal to the surface. The calculated $\mathrm{Si}-\mathrm{Si}$ bond lengths from the PBE and DFT-D schemes are 2.284 and $2.277 \AA$, respectively. They are in excellent agreement with previous theoretical result and the experimental value of $2.2 \pm 0.1 \AA$. The theories also reproduce the experimental lattice constant [14] of the unit cell of pristine silicene very well.

We considered the STW defect in silicene. The geometric structures of the defect were fully relaxed without symmetry information. The obtained lattice constants, formation energy and work functions (WF) are $22.787 \AA$, $1.93 \mathrm{eV}$, and $4.95 \mathrm{eV}$, respectively. Compared with their graphene analogues, the STW defect of silicene are thermodynamically more stable based on the analogous definition of formation energy and chemical potential of the atoms, and therefore are more easily to be formed.

\section{B. Adsorption of a Lithium Ion}

To save computation, we only consider the adsorption of a lithium atom on the sites with a relatively strong binding. Figure 1 demonstrates some main adsorption sites of a lithium atom on pristine and defective silicenes. For pristine silicene, the lithium atoms bind most strongly to the hollow (H) site, and the second favorable adsorption site is the valley (V) site on top of the lower silicon atoms. The binding energies of the lithium adatom at the $\mathrm{H}$ and $\mathrm{V}$ sites are 0.84 and $0.63 \mathrm{eV}$, respectively. The distances between the adatom and the nearest silicon atom are 2.68 and $2.64 \AA$ for the $\mathrm{H}$ and $\mathrm{V}$ sites, respectively. If the cohesive energy per lithium atom in the bcc crystal ( $1.28 \mathrm{eV}$ from the DFT-D scheme) is taken in consideration, we reproduce the theoretical results of Lin and $\mathrm{Ni}$ [15] very well. In their work, the binding energy in terms of the energy of an isolated lithium atom is 2.129 and $1.896 \mathrm{eV}$ at the $\mathrm{H}$ and $\mathrm{V}$ sites, respectively. 


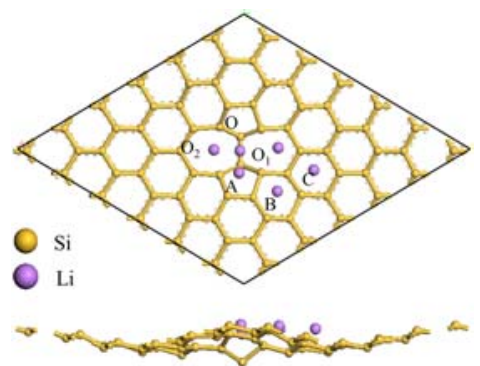

Figure 1. Main adsorption sites of a lithium atom on the STW defective silicene.

There are six kinds of adsorption sites shown in Figure 1 for the defective silicenes. We label the sites on the top of the defects as $\mathrm{O}, \mathrm{O}_{1}$ or $\mathrm{O}_{2}$, and those on top of the hexagonal rings next to the defects as $\mathrm{A}, \mathrm{B}$, or $\mathrm{C}$. The $\mathrm{O}$ site is usually located at the place nearest to the center of the defect.

TABLE I. Binding ENERgies, Charge transfer $(Q)$ AND the NEAREST DISTANCE BETWEEN LI AND SI ATOMS AT OK

\begin{tabular}{|l|l|l|l|l|l|l|}
\hline \multirow{2}{*}{ Site } & \multicolumn{2}{|c|}{$\boldsymbol{E}_{\mathbf{a}}(\mathbf{e V})$} & \multicolumn{2}{c|}{$\boldsymbol{L}_{\text {min,Li-Si }}(\AA)$} & \multicolumn{2}{c|}{$Q(\mathbf{e})$} \\
\cline { 2 - 7 } & \multicolumn{7}{|c|}{ Pristine silicene } \\
\hline $\mathrm{H}$ & -0.05 & 0.84 & 2.74 & 2.68 & 0.271 & 0.250 \\
\hline $\mathrm{V}$ & -0.20 & 0.63 & 2.66 & 2.64 & 0.333 & 0.321 \\
\hline \multicolumn{7}{|c|}{ STW defect } \\
\hline $\mathrm{O}$ & 0.32 & 1.22 & 2.72 & 2.69 & 0.248 & 0.195 \\
\hline $\mathrm{O}_{1}$ & 0.24 & 1.13 & 2.68 & 2.66 & 0.228 & 0.232 \\
\hline $\mathrm{O}_{2}$ & 0.12 & 1.02 & 2.69 & 2.75 & 0.256 & 0.216 \\
\hline $\mathrm{A}$ & 0.18 & 0.98 & 2.64 & 2.70 & 2.87 & 0.280 \\
\hline B & 0.09 & 0.94 & 2.73 & 2.68 & 2.65 & 0.246 \\
\hline $\mathrm{C}$ & 0.05 & 0.92 & 2.73 & 2.70 & 2.68 & 0.248 \\
\hline
\end{tabular}

The binding energies, charge transfers and geometrical parameters from the PBE and DFT-D schemes are listed in Table I. Both the PBE and DFT-D schemes predict that the binding energies of a lithium atom adsorbed on all sites in the defective silicenes are larger than that in pristine silicene. The binding energies of a lithium atom on a STW defective silicene nanosheet are greater than those on pristine silicone. Both schemes predict that the $\mathrm{H}$ site in pristine and $\mathrm{O}$ site in the STW are the most preferred adsorption site. In most cases, the distance between the lithium atom and the nearest silicon atom, $l_{\text {min,Li-Si, }}$, predicted from the DFT-D scheme is shorter than that from the PBE scheme due to the attractive $\mathrm{vdW}$ interactions. However, when the lithium atom is adsorbed on the $\mathrm{O}_{2}$ and $\mathrm{A}$ sites in the STW defect, the dispersion correction makes the nearest distance $l_{\text {min,Li-Si }}$ increase by $0.02-0.06 \AA$. Compared with the adsorption structures on these sites predicted from the PBE scheme, the silicon atoms interacting with the lithium atom have rearranged during the geometric optimization using the DFTD scheme. Overall, the vdW interactions may change the nearest distance $l_{\min , \mathrm{Li}-\mathrm{Si}}$ by a value between -0.05 and +0.06 $\AA$. Comparison of the data listed in Table I we can infer that the vdW interactions have little effect on the nearest distance $l_{\text {min,Li-Si }}$ and the charge transfer, and can strengthen the binding between the lithium atom and the monolayer pristine or defective silicene.

\section{Diffusion on and through Silicenes}

Considering the importance of lithium mobility in improving the charge/discharging performance of lithiumion batteries, we investigate the energy profiles of a lithium atom migrating from one site to a neighboring site on and through monolayer pristine and STW defective silicenes. As shown in Figure 2, the calculated energy barrier for diffusion from the $\mathrm{H}$ site to the $\mathrm{V}$ site in pristine silicene is $0.27 \mathrm{eV}$, which is lower than the diffusion barrier of $0.327 \mathrm{eV}$ for a lithium atom migrating on pristine graphene. Considering the difference in lithium concentration, our calculated diffusion barrier for a lithium atom moving from the $\mathrm{H}$ site to the $\mathrm{V}$ site agrees well with the theoretical result obtained by Tritsaris et al. [7].

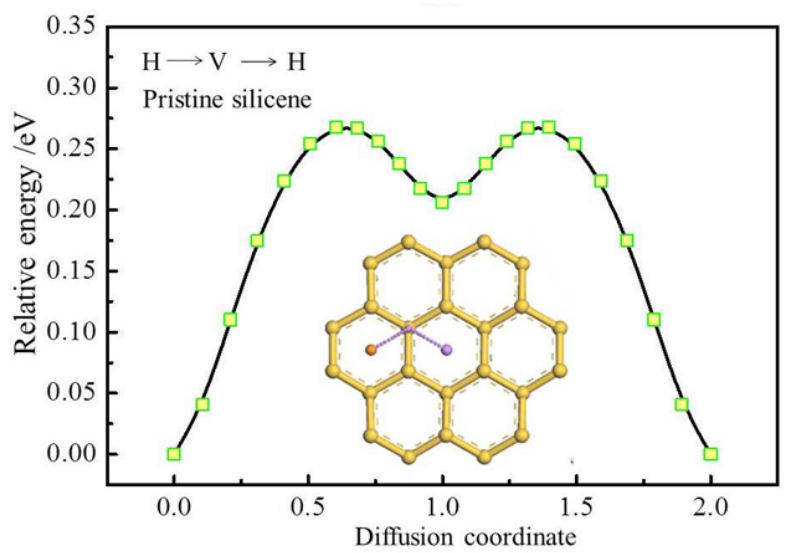

Figure 2. Diffusion path and relative energy profile of a lithium atom on pristine silicene from the $\mathrm{H}$ site to the $\mathrm{V}$ site and then to the next $\mathrm{H}$ site.

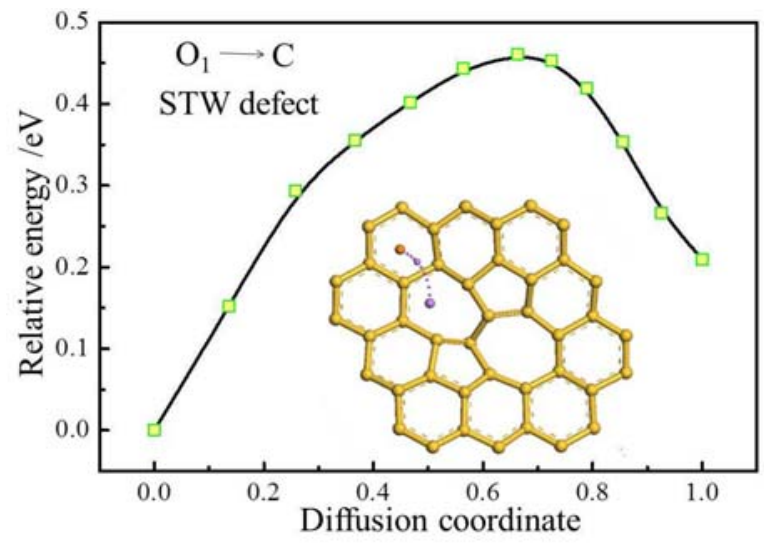

Figure 3. Relative energy profile and diffusion path for a lithium atom moving from the $\mathrm{O}_{1}$ site to the $\mathrm{C}$ site on a STW defect.

Figure 2 depicts the relative energy profile for lithium atom diffusion from the site $\mathrm{O}_{1}$ to the site $\mathrm{C}$ in the STW 
defective silicone. The barrier is $0.46 \mathrm{eV}$. Our DFT-D results show that the lithium atom migrating along other diffusion paths $\left(\mathrm{O} \rightarrow \mathrm{O}_{1}, \mathrm{O} \rightarrow \mathrm{O}_{2}, \mathrm{O} \rightarrow \mathrm{A}, \mathrm{O}_{1} \rightarrow \mathrm{B}\right)$ are easier than that along the site $\mathrm{O}_{1} \rightarrow \mathrm{C}$. Among the different diffusion paths, the $\mathrm{O} \rightarrow \mathrm{O}_{2}$ path is with the lowest diffusion barrier on the STW defect. We found that vdW interactions may either increase or decrease the energy barrier, depending on both the TS and initial state structures. vdW interactions have significant contributions to the diffusion barriers, but in some cases the $\mathrm{O}_{1}-\mathrm{C}$ diffusion paths on the STW defect, vdW interaction contributions are only marginal, inferring that $\mathrm{vdW}$ interaction contributions to the energies of the initial and TS states appear to cancel each other out.

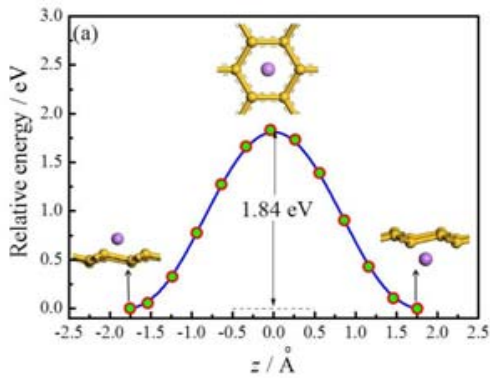

Figure 4. Energy changes of a lithium atom diffusing through pristine silicenes.

The DFT-D results in Figure 3 show that a diffusion barrier of $1.84 \mathrm{eV}$ is required for a lithium atom migrating through a hexagon of silicene. This value is substantially lower than that through graphene. From the calculations we found that a lithium atom moving through the STW defects needs to overcome the energy barriers of $0.56 \mathrm{eV}$. Comparison with the results calculated from the PBE functional shows that vdW interactions contribute +0.25 , and $+0.04 \mathrm{eV}$ to the diffusion barriers of a lithium atom migrating through the pores in pristine and STW defective silicenes, respectively. It seems to be true that $\mathrm{vdW}$ interactions contribute more to diffusion barriers of a lithium atom migrating through the narrower pores. For the STW defect, vdW interactions have a small contribution to the diffusion barrier due to the marginal effect of vdW forces on the large symmetrical pores. The calculated results indicate that the lithium atom moves more easily through pristine and defective silicenes than through their graphene counterpart.

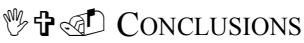

$A b$ initio density functional theory was employed to investigate the adsorption and diffusion of a lithium atom on pristine and STW defective silicenes. Both PBE and DFT-D schemes were used in order to explore the role of $\mathrm{vdW}$ interactions in adsorption and diffusion on and through the pore in pristine and defective silicenes. The binding energies of a lithium atom on the most favorable adsorption sites in pristine and STW defective silicenes are 0.84 and $1.22 \mathrm{eV}$, respectively. It is difficult for a lithium atom migrating through pristine silicene due to high diffusion barriers. In contrast, the lithium atom moves fast through the STW defect.
The vdW interactions play a crucial role in the lithium adsorption. In most cases they contribute $0.78-0.99 \mathrm{eV}$ to the binding energies of a lithium atom adsorbed on pristine and STW defective silicenes, and this indicates that the interaction of the lithium atom with the silicene substrate is a strong physisorption. The vdW interactions can cause either an increase or a decrease in the diffusion barriers dependent on the competition of their contributions to the energies between the TS and initial structures. The vdW interactions have a great contribution to diffusion barrier of a lithium atom migrating through a narrow pore in the silicene substrates. But in the case of the defective silicene with a large symmetric pore like those in the STW defects, vdW interactions only play a marginal role due to that a relative long distance between the lithium atom and the silicon atoms reduces the vdW interactions and most of them cancel each other out.

Compared with their analogue graphene, the enhanced binding energies and comparable or lowered diffusion barriers for a lithium atom moving on and through the silicene substrates can be found. These results support the potential application of STW-defective silicene nanosheets as anode materials of high-rate and high capacity lithium-ion batteries.

\section{ACKNOWLEDGMENT}

We are greatly indebted to Ms. Yong-Jun Du for her efforts in the preparation of the manuscript. We also gratefully acknowledge financial support from the National Natural Science Foundation of China (grant no. 21376131).

\section{REFERENCES}

[1] U. Kasavajjula, C. Wang, and A. J. Appleby, "Nano- and bulksilicon-based insertion anodes for lithium-ion secondary cells," J. Power Sources, vol. 163, pp. 1003-1039, 2007.

[2] H. Wu, and Y. Cui, "Designing nanostructured Si anodes for high energy lithium ion batteries," Nano Today, vol. 7, pp. 414-429, 2012..

[3] T.L. Chan, and J.R. Chelikowsky, "Controlling diffusion of lithium in silicon nanostructures," Nano Lett., vol. 10, pp. 821-825, 2010.

[4] Y.X. Yu, "Can all nitrogen-doped defects improve the performance of graphene anode materials for lithium-ion batteries," Phys. Chem. Chem. Phys., vol. 15, pp. 16819-16827, 2013.

[5] H. Oughaddou, H. Enriquez, H. Yildirim, A. J. Mayne, A. Bendounan, G. Dujardin, M. A. Ali, and A. Kara, "Silicene: a promising new 2D material," Prog. Surf. Sci., vol. 90, pp. 46-83, 2015

[6] T.H. Osborn, and A.A. Farajian, "Stability of lithiated silicene from first principles," J. Phys. Chem. C, vol. 116, pp. 22916-22920, 2012.

[7] G.A. Tritsaris, E. Kaxiras, S. Meng, and E. Wang, "Adsorption and diffusion of lithium on layered silicon for Li-ion storage," Nano Lett., vol. 13, pp. 2258-2263, 2013.

[8] H. Sahin, J. Sivek, S. Li, B. Partoens, and F. M. Peeters, "StoneWales defects in silicene: formation, stability, and reactivity of defect sites,” Phys. Rev. B, vol. 88, art. No. 045434, 2013.

[9] S. Grimme, "Semiempirical GGA-type density functional constructed with a long-range dispersion correction," J. Comput. Chem., vol. 27, pp. 1787-1799, 2006. 
[10] Y.X. Yu, "Graphenylene: A promising anode material for lithium-ion batteries with high mobility and storage," J. Mater. Chem. A, vol. 1, pp. 13559-13566, 2013.

[11] B. Delley, "Calculation of electrostatics in crystals and large molecules," J. Phys. Chem., vol. 100, pp. 6107-6110, 1996.

[12] B. Delley, "From molecules to solids with the Dmol ${ }^{3}$ approach," J. Chem. Phys., vol. 113, pp. 7756-7764, 2000.
[13] G. Henkelman, and H. Jonsson, "Improved tangent estimate in the nudged elastic band method for finding energy paths and saddle points," J. Chem. Phys., vol. 113, pp. 9978-, 2000.

[14] P. Vogt, P. De Padova, C. Quaresima, J. Avila, E. Frantzeskakis, M. C. Asensio, A. Resta, B. Ealet, and G. L. Lay, "Silicene: compelling experimental evidence for graphene like two-dimensional silicon," Phys. Rev. Lett, vol. 108, art. no. 155501, 2012.

[15] X.Q. Lin, and J. Ni, "Much stronger binding of matal adatoms to silicene than to graphene: a first-principles study," Phys. Rev. B, vol. 86, art. no. $075440,2012$. 